\title{
SOUSLIN'S HYPOTHESIS AND CONVERGENCE IN CATEGORY
}

\author{
ARNOLD W. MILLER \\ (Communicated by Franklin D. Tall)
}

\begin{abstract}
A sequence of functions $f_{n}: X \rightarrow \mathbb{R}$ from a Baire space $X$ to the reals $\mathbb{R}$ is said to converge in category iff every subsequence has a subsequence which converges on all but a meager set. We show that if there exists a Souslin tree, then there exists a nonatomic Baire space $X$ such that every sequence which converges in category converges everywhere on a comeager set. This answers a question of Wagner and Wilczynski who proved the converse.
\end{abstract}

Suppose that $S \subseteq P(X)$ is a $\sigma$-field of subsets of $X$ and $I \subseteq S$ is a $\sigma$-ideal. If $I$ has the countable chain condition (ccc), i.e., every family of disjoint sets in $S \backslash I$ is countable, then $S / I$ is a complete boolean algebra. A boolean algebra is atomic iff there is an atom beneath every nonzero element.

A function $f: X \rightarrow \mathbb{R}$ is $S$-measurable iff $f^{-1}(U) \in S$ for every open set $U$. A sequence of $S$-measurable functions $f_{n}: X \rightarrow \mathbb{R}$ converges $I$-a.e. to a function $f$ iff there exists $A \in I$ such that $f_{n}(x) \rightarrow f(x)$ for all $x \in(X \backslash A)$. If $(X, S, \mu)$ is a finite measure space, then a sequence of measurable functions $f_{n}: X \rightarrow \mathbb{R}$ converges in measure to a function $f$ iff for any $\epsilon>0$ there exists $N$ such that for any $n>N$ :

$$
\mu\left(\left\{x \in X:\left|f_{n}(x)-f(x)\right|>\epsilon\right\}\right)<\epsilon .
$$

In this case if $I$ is the ideal of measure zero sets, then $f_{n}$ converges to $f$ in measure iff every subsequence $\left\{f_{n}: n \in A\right\}$ (where $A \subseteq \mathbb{N}$ ) has a subsequence $B \subseteq A$ such that $\left\{f_{n}: n \in B\right\}$ converges $I$-a.e. This allows us to define convergence in measure without mentioning the measure, only the ideal $I$. So in the abstract setting define the following: $f_{n}$ converges to $f$ with respect to $I$ iff every subsequence $\left\{f_{n}: n \in A\right\}$ has a subsequence $B \subseteq A$ such that $\left\{f_{n}: n \in B\right\}$ converges $I$-a.e. (where $A$ and $B$ range over infinite sets of natural numbers.) For more background on this subject in case $I$ is the ideal of meager sets, see Poreda, Wagner-Bojakoska, and Wilczyński [PWW] and Ciesielski, Larson, and Ostaszewski [CLO].

Marczewski $[\mathrm{M}]$ showed that if $(X, S, \mu)$ is an atomic measure and $I$ the $\mu$-null sets, then ' $I$-a.e. convergence' is the same as 'convergence with respect to $I$ '.

Gribanov [G] proved the converse, if $(X, S, \mu)$ is a finite measure space and $I$ the $\mu$-null sets, then if ' $I$-a.e. convergence' is the same as 'convergence with respect to $I$ ' then $\mu$ is an atomic measure.

Received by the editors November 2, 1994.

1991 Mathematics Subject Classification. Primary 28A20; Secondary 03E65, 54E52.

I want to thank Krzysztof Ciesielski for many helpful conversations.

The results presented in this paper were obtained during the Joint US-Polish Workshop in Real Analysis, Łódź, Poland, July 1994. The workshop was partially supported by the NSF grant INT-9401673. 
Souslin's Hypothesis ( $\mathrm{SH})$ is the statement that there are no Souslin lines. It is known to be independent (see Solovay and Tennenbaum [ST]). It was the inspiration for Martin's Axiom.

Theorem 1 (Wagner and Wilczyński [WW]). Assume $S H$. Then for any $\sigma$-field $S$ and ccc $\sigma$-ideal $I \subseteq S$ the following are equivalent:

- 'I-a.e. convergence' is the same as 'convergence with respect to I' for $S$ measurable sequences of real-valued functions, and

- the complete boolean algebra $S / I$ is atomic.

At the real analysis meeting in Łódź, Poland in July 1994, Wilczyński asked whether or not $\mathrm{SH}$ is needed for the Theorem above. We show here that the conclusion of Theorem 1 implies Souslin's Hypothesis.

Theorem 2. Suppose SH is false (so there exists a Souslin tree). Then there exists a regular topological space $X$ such that

1. $X$ has no isolated points,

2. $X$ is ccc (every family of disjoint open sets is countable),

3. every nonempty open subset of $X$ is nonmeager, and

4. if $I$ is the $\sigma$-ideal of meager subsets of $X$, then ' $I$-a.e. convergence' is the same as 'convergence with respect to I' for any sequence of Baire measurable real-valued functions.

Hence if $S$ is the $\sigma$-ideal of sets with the property of Baire and I the $\sigma$-ideal of meager sets, then $S / I$ is ccc and nonatomic, but the two types of convergence are the same.

Proof. Define $(T,<)$ to be an $\omega_{1}$-tree iff it is a partial order and for each $s \in T$ the set $\{t \in T: t<s\}$ is well-ordered by $<$ with some countable order type, $\alpha<\omega_{1}$. We let

$$
T_{\alpha}=\{s \in T:\{t \in T: t<s\} \text { has order type } \alpha\} .
$$

Also

$$
T_{<\alpha}=\bigcup\left\{T_{\beta}: \beta<\alpha\right\} .
$$

Define $C \subseteq T$ is a chain iff for every $s, t \in C$ either $s \leq t$ or $t \leq s$.

Define $A \subseteq T$ is an antichain iff for any $s, t \in A$ if $s \leq t$, then $s=t$, i.e. distinct elements are $\leq$-incomparable.

Define $T$ is a Souslin tree iff $T$ is an $\omega_{1}$-tree in which every chain and antichain is countable. (Note that since $T_{\alpha}$ is an antichain, it must be countable.)

$\mathrm{SH}$ is equivalent to saying there is no Souslin tree. Every Souslin tree contains a normal Souslin tree, i.e., a Souslin tree $T$ such that for every $\alpha<\beta<\omega_{1}$ and $s \in T_{\alpha}$ there exists a $t \in T_{\beta}$ with $s<t$. (Just throw out nodes of $T$ which do not have extensions arbitrarily high in the tree.) For more on Souslin trees see Todorčevič $[\mathrm{T}]$.

Now we are ready to define our space $X$. Let the elements of $X$ be maximal chains of $T$. For each $s \in T$ let

$$
C_{s}=\{b \in X: s \in b\}
$$

and let

$$
\left\{C_{s}: s \in T\right\}
$$


be an open basis for the topology on $X$. Note that $C_{s} \cap C_{t}$ is either empty or equal to either $C_{s}$ or $C_{t}$ depending on whether $s$ and $t$ are incomparable, or $t \leq s$ or $s \leq t$, respectively. Each $C_{s}$ is clopen since its complement is the union of $C_{t}$ for $t$ which are incomparable to $s$. $X$ has no isolated points, since given any $s \in T$ there must be incomparable extensions of $s$ (because $T$ is normal) and therefore at least two maximal chains containing $s$, so $C_{s}$ is not a singleton. Clearly $X$ has the countable chain condition.

Lemma 3. Open subsets of $X$ are nonmeager. In fact, the intersection of countably many open dense sets contains an open dense set.

Proof. The proof is quite standard and can be found in the reference books: Kunen $[\mathrm{K}]$ or Jech $[\mathrm{J}]$. For the convenience of the reader we include it.

Suppose $\left(U_{n}: n \in \omega\right)$ is a sequence of open dense subsets of $X$. Let $A_{n} \subseteq T$ be an antichain which is maximal with respect to the property that $C_{s} \subseteq U_{n}$ for each $s \in A_{n}$. Since $U_{n}$ is open dense in $X, A_{n}$ will be a maximal antichain in $T$.

Let

$$
V_{n}=\bigcup\left\{C_{s}: s \in A_{n}\right\} .
$$

Then $V_{n} \subseteq U_{n}$ and $V_{n}$ is open dense. (It is dense, because given any $C_{t}$ there exist $s \in A_{n}$ and $r \in T$ with $t \leq r$ and $s \leq r$, hence $C_{r} \subseteq V_{n} \cap C_{t}$.)

Choose $\alpha<\omega_{1}$ so that for each $n \in \omega$ the (necessarily countable) antichain $A_{n} \subseteq T_{<\alpha}$. Let

$$
U=\bigcup\left\{C_{s}: s \in T_{\alpha}\right\}
$$

Note that since $T$ is normal, $U$ is an open dense set. Also

$$
U \subseteq \bigcap_{n<\omega} V_{n} \subseteq \bigcap_{n<\omega} U_{n}
$$

$U \subseteq V_{n}$ because for any $b \in U$ if $b \in C_{s}$ for some $s \in T_{\alpha}$ there must be $t \in A_{n}$ comparable to it, since $A_{n}$ is a maximal antichain, and since $A_{n} \subseteq T_{<\alpha}$, it must be that $t<s$ and so $b \in C_{t} \subseteq V_{n}$.

Lemma 4. Suppose $f: X \rightarrow \mathbb{R}$ is a real-valued Baire function. Then there exists $\alpha<\omega_{1}$ such that for each $s \in T_{\alpha}$ the function $f$ is constant on $C_{s}$.

Proof. Let $\mathcal{B}$ be a countable open basis for $\mathbb{R}$. For each $B \in \mathcal{B}$ the set $f^{-1}(B)$ has the property of Baire (open modulo meager). So there exists an open $U_{B}$ such that

$$
U_{B} \Delta f^{-1}(B) \text { is meager. }
$$

By the proof of Lemma 3 we may assume that

$$
U_{B}=\bigcup\left\{C_{s}: s \in A_{B}\right\} .
$$

By the proof of Lemma 3 there exists an $\alpha<\omega_{1}$ such that

- each $A_{B} \subseteq T_{<\alpha}$ and

- if $U$ is the open dense set $\bigcup\left\{C_{s}: s \in T_{\alpha}\right\}$, then $U$ is disjoint from $U_{B} \Delta f^{-1}(B)$ for each $B \in \mathcal{B}$.

But now, $f$ is constant on each $C_{s}$ for $s \in T_{\alpha}$. Otherwise, suppose that $f(b) \neq f(c)$ for some $b, c \in C_{s}$ for some $s \in T_{\alpha}$. Then suppose that $f(b) \in B$ and $f(c) \notin B$ for some $B \in \mathcal{B}$. Because $b \in\left(f^{-1}(B) \cap U\right)$ and $U$ is disjoint from $U_{B} \Delta f^{-1}(B)$, it must be that $b \in U_{B}$. Hence there exists $t \in T_{<\alpha}$ such that $C_{t} \subseteq U_{B}$ and $b \in C_{t}$. Since $t<s$, it must be that $c \in C_{t}$ and so $c \in f^{-1}(B)$, which contradicts $f(c) \notin B$. 
Steprańs $[\mathrm{S}]$ shows that every continuous function on a Souslin tree takes on only countably many values.

Lemma 5. Suppose $\left\{f_{n}: X \rightarrow \mathbb{R}: n \in \omega\right\}$ is a countable set of real-valued Baire functions. Then there exists $\alpha<\omega_{1}$ such that for each $s \in T_{\alpha}$ and $n<\omega$ the function $f_{n}$ is constant on $C_{s}$.

Proof. Apply Lemma 4 countably many times and take the supremum of the $\alpha_{n}$.

Finally, we prove the theorem. The idea of the proof is to use the argument of the atomic case, where the 'atoms' are supplied by Lemma 5 . Since ' $I$-a.e. convergence' always implies 'convergence with respect to $I$ ', it is enough to see the converse. So let $f_{n}: X \rightarrow \mathbb{R}$ be Baire functions which converge to $f: X \rightarrow \mathbb{R}$ with respect to $I$, i.e. every subsequence has a subsequence which converges on a comeager set to $f$. By Lemma 5 there exists $\alpha<\omega_{1}$ such that for each $s \in T_{\alpha}$ and $n<\omega$ the function $f_{n}$ is constant on $C_{s}$. Since every subsequence has a convergent subsequence, it must be that for each fixed $s \in T_{\alpha}$ the constant values of $f_{n}$ on $C_{s}$ converge to a constant value. It follows that the sequence $f_{n}(x)$ converges to $f(x)$ on the dense open set $\left\{C_{s}: s \in T_{\alpha}\right\}$.

\section{REFERENCES}

[CLO] K.Ciesielski, L.Larson, and K.Ostaszewski, I-Density Continuous Functions, Memoirs of the American Mathematical Society, 107(1994). MR 94f:54035

[G] J.M.Gribanov, Remark on convergence a.e. and convergence in measure, Comm. Math. Univ Carolinae, 7(1966), 297-300. MR 34:1482

[K] K.Kunen, Set Theory , North-Holland, 1980. MR 82f:03001

[J] T.Jech, Set Theory, Academic Press, 1978. MR 80a:03062

[PWW] W.Poreda, E.Wagner-Bojakoska, and W.Wilczyński, A category analogue of the density topology, Fundamenta Mathematicae, 125(1985), 167-173. MR 87b:54034

[M] E. Marczewski, Remarks on the convergence of measurable sets and measurable functions, Colloquium Mathematica, 3 (1955), 118-124. MR 16:1092f

[S] J.Steprańs, Trees and continuous mappings into the real line, Topology and Its Applications, 12(1981), 181-185. MR 83a:54014

[ST] R.M.Solovay, S.Tennenbaum, Iterated Cohen extensions and Souslin's problem, Annals of Mathematics, 94(1971), 201-245. MR 45:3212

[T] S.Todorčevič, Trees and linearly ordered sets, in Handbook of Set Theoretic Topology, North-Holland, (1984), 235-293. MR 86h:54040

[WW] E.Wagner, W.Wilczyński, Convergence of sequences of measurable functions, Acta Mathematica Academiae Scientiarum Hungaricae, 36(1980), 125-128. MR 82c:28014

University of Wisconsin-Madison, Department of Mathematics, Van Vleck Hall, 480 Lincoln Drive, Madison, Wisconsin 53706-1388

E-mail address: miller@math.wisc.edu 